\title{
Electron correlation effects and magnetic ordering at the $\operatorname{Gd}(0001)$ surface
}

\author{
A.B. Shick, W.E. Pickett and C. S. Fadley \\ Department of Physics, University of California, Davis, CA 95616
}

\begin{abstract}
Effects of electron correlation on the electronic structure and magnetic properties of the $\mathrm{Gd}(0001)$ surface are investigated using of the full-potential linearized augmented plane wave implementation of correlated band theory ("LDA+U"). The use of LDA+U instead of LDA (local density approximation) total energy calculations produces the correct ferromagnetic ground state for both bulk Gd and the Gd surface. Surface strain relaxation leads to an $90 \%$ enhancement of the interlayer surface-to-bulk effective exchange coupling. Application of a Landau-Ginzburg type theory yields a $30 \%$ enhancement of the Curie temperature at the surface, in very good agreement with the experiment.
\end{abstract}

Many of the magnetic properties of Gd metal are well understood [1]. The half-filled 4 f-shell $\left(S=\frac{7}{2}, L=0\right)$ of Gd leads to a formation of a well localized spin-only magnetic moment. These localized spin moments couple through an RKKY-type exchange interaction to form a ferromagnetic (FM) Heisenberg system with a bulk Curie temperature $\left(T_{c}^{b}\right)$ of $293 \mathrm{~K}$ [1]. FM order polarizes the conduction electrons and leads to a total magnetic moment of $7.63 \mu_{B} / \mathrm{Gd}$ atom [2].

However, in spite of relatively simple bulk magnetic behavior, the magnetism of the Gd surface is rather unusual [3]. The results of different spectroscopic measurements suggest a significant enhancement of the surface Curie temperature $\left(T_{c}^{s}\right)$ for $\operatorname{Gd}(0001)$. Gd is thus one of only three ferromagnets (including $\mathrm{Tb}$ and $\mathrm{FeNi}_{3}$ ) for which such an increased $T_{c}^{s}$ has been observed [1]. After a first observation of this effect in Gd by Rau et al. [4], Weller et al. verified it for $400 \AA$ thick Gd(0001) films grown on a W(110) substrate, by comparing spin-polarized low energy electron diffraction (LEED) and the magneto-optic Kerr effect measurements [5], and further suggested a possible anti-ferromagnetic (AFM) alignment of the surface layer(s) with respect to the bulk FM Gd. Further investigations with spin-polarized valence and core photoemission spectroscopy (PES) [6] did not confirm an existence of this surface AFM coupling. Instead, the in-plane component of surface layer magnetization was observed to be parallel to the bulk [6 8], althrough the possibility of canted or mixed in-plane and out-of-plane surface magnetic ordering was suggested. Very recent spin-polarized photoelectron diffraction experiments 9 for bulklike $\approx$ $300 \AA$ thick epitaxial Gd/W(110) films clearly indicate temperature dependent core level spin asymmetries well above the bulk $T_{c}^{b}$, also suggesting surface enhancement of $T_{c}^{s}$ of as much as $85 \mathrm{~K}$. However, to date, there has been no quantitative theoretical explanation for this enhanced $T_{c}^{s}$.

The aim of this paper is to show that first principles calculations which account for both electronic correlations of the $4 \mathrm{f}$ electrons and the relaxation of the surface atomic positions can provide such a quantitative description of the electronic and magnetic structure of the $\operatorname{Gd}(0001)$ surface. Using the results of total energy cal- culations and the Landau-Ginzburg model we show that there is an increase of $T_{c}$ at the Gd surface due to an enhanced surface-to-bulk effective exchange coupling that is in turn caused by the surface structure relaxation. We emphasize the substantial role of electron correlations for the Gd f-electrons to obtain a correct FM ground state for both bulk Gd and the Gd surface. We also emphasize the role of structure relaxation as a "driving force" for the $T_{c}$ enhancement.

1.Difficulties of local (spin) density approximation (LDA) and generalized gradient approximation (GGA) and limitations of a core treatment for $G d$ if electrons. Since the pioneering work of Dimmock and Freeman 10 there have been other attempts to describe the $4 \mathrm{f}$ states of Gd in terms of the localized "4f-core" electron model [11] (in which the $4 \mathrm{f}$ states of Gd are treated as a part of fully localized atomic core). Singh [12] performed a detailed analysis of the limitations of this "4f-core" model and achieved very good agreement with experiment for the ground state lattice constant and the magnetic moment of FM bulk Gd by using a "4f-band" model and LDA, but he did not consider a possible AFM phase. Recent full potential linear muffin-tin orbitals (LMTO) calculations 13,14 show that both the LDA and the GGA yield an AFM phase that is lower in energy than the FM phase. This problem is solved by employing the $\mathrm{LDA}+\mathrm{U}$ [15] method to treat the electron correlations for the $4 \mathrm{f}$-electrons of $\mathrm{Gd}$. It was demonstrated [13] that the use of LDA+U [15] instead of LDA yields a correct FM ground state and also provides 4 f electron binding energies in good agreement with experiment. Recently we confirmed quantitatively the conclusions of Ref. 13. for bulk Gd using the LDA+U total energy functional with the full-potential linearized augmented plane wave (FLAPW) method [16].

The first total energy FLAPW calculations of the magnetic ordering at the Gd surface [17] using the 4f-core model reported an AFM coupling of the surface layer with respect to the bulk, in agreement with early interpretation of experimental data [5]. The full potential LMTO calculations using the $4 \mathrm{f}$-core model [14 did not reproduce the results of Ref. [17] and yielded FM coupling between the surface and bulk magnetization, in 
agreement with the most recent experiments [6]. It is thus clear that: (i) the $4 \mathrm{f}$-band model with LDA fails to account for the correct magnetic ordering for bulk $\mathrm{Gd}$, and the use of GGA instead of LDA does not improve the situation; (ii) two LDA 4f-core model calculations with LDA yield conflicting results ( [14] and [17]) for the magnetic ground state at the Gd surface.

Since the LDA $+\mathrm{U}$ method works well to describe the electronic structure and magnetic ground state for bulk Gd, we decided to apply it in electronic structure calculations and total energy determinations of the magnetic behavior of the Gd surface.

2. Computational results. The FLAPW method 18 is employed to perform scalar-relativistic self-consistent film calculations for Gd. The fully relativistic selfconsistent version 19] of this method is then used to perform the final LDA calculations. The $\mathrm{LDA}+\mathrm{U}$ calculations are based on the scalar-relativistic version of the FLAPW method [16]. The literature values [13] of the on-site repulsion $U=6.7 \mathrm{eV}$ and exchange $J=0.7 \mathrm{eV}$ were used in the calculations.

For the $\operatorname{Gd}(0001)$ surface, we choose the isolated slab model based on 7-layer Gd film (with z-reflection symmetry) and in the first set of calculations use the bulk lattice constant (3.634 $\AA$ ) and c/a ratio (1.587) 20]. Here, 32 special $\mathrm{k}$-points [21] in the irreducible $1 / 3$ part of the 2D BZ 22 were used, with Gaussian smearing for k-points weighting. The "muffin-tin" radius values of $R_{M T}=3.2$ a.u. and $R_{M T} \times K_{\max }=9.6$ (where, $K_{\max }$ is the cut-off for LAPW basis set) were used.

2A.LDA results. The spin magnetic moments for a Gd film with its surface layer magnetically coupled parallel $(\uparrow \uparrow)$ and antiparallel $(\downarrow \uparrow)$ to the FM bulk resulting from the scalar-relativistic LDA calculations show that the magnetically active $f$ states are almost fully polarized (with the magnetic moment $6.88 \mu_{B}$ in the bulk and $6.82 \mu_{B}$ at the surface) and induced spin polarization of $\approx 0.5 \mu_{B}$ /atom of conduction electrons (mainly d states). This magnetic coupling is a result of intraatomic interband exchange interaction between conduction band and localized $\mathrm{f}$ electrons as incorporated in the s-f exchange model [23] and can be understood to be due to a positive inter-band $d-f$ exchange coupling 24. There is a slight decrease of the $4 \mathrm{f}$ magnetic moment at the surface layer due to an increase of minority spin 4 f occupation.

Starting from the results of scalar-relativistic calculations, we then performed self-consistent relativistic LDA calculations for a Gd-film, assuming [0001] spin axis direction. The spin moments are slightly decreased for $\mathrm{f}$ states in comparison with scalar-relativistic calculations, due to an increase of minority spin occupation of the 4 states. The small spin-orbit induced orbital magnetic moments $\left(0.14 \mu_{B}\right.$ for the bulk and $0.33 \mu_{B}$ for the surface atoms) are mainly due to the $4 \mathrm{f}$ minority spin contribution. A parallel coupling between spin and orbital moments for $4 \mathrm{f}$ states is consistent with the 3rd Hund rule. The orbital moments from $5 \mathrm{~d}$ states are about $0.02 \mu_{B}$ per Gd atom and coupled anti-parallel to the spin mo- ments, again consistent with the 3rd Hund rule. The values of total magnetic moment (the sum of spin and orbital moments) are close to the values of spin moment from scalar-relativistic calculations.

The total energy difference $\Delta E_{(\downarrow \uparrow-\uparrow \uparrow)}$ between the two surface magnetic configurations $\uparrow \uparrow$ and $\downarrow \uparrow$ defined above is positive (36 meV/atom in scalar-relativistic calculations) and does not change appreciably when spin-orbit coupling is included (40 meV/atom). The effect of the spin-orbit interaction is seen to be very small for the energetics of $\mathrm{Gd}$ due to the fact that the $4 \mathrm{f}$ spin-majority band is fully occupied and the $4 \mathrm{f}$ spin-minority band is almost empty. Therefore, the spin-orbit coupling does not affect the calculated values of magnetic and total energy properties of $\mathrm{Gd}$ and does not assist in resolving the limitations of LDA.

2B. $L D A+U$ results. The spin magnetic moments for a Gd film with surface layer magnetically coupled parallel to the FM bulk resulting from the scalar-relativistic $\mathrm{LDA}+\mathrm{U}$ calculations are shown in Table II. There is a moderate enhancement of the magnetic moment of $4 \mathrm{f}$ states compared to LDA values $\left(\approx 0.1 \mu_{B}\right.$ /atom) due to an upward shift of $1.5 \mathrm{eV}$ of minority spin $4 \mathrm{f}$ states. There is practically no difference between surface and bulk f state magnetic moments. The total magnetic moment at the surface layer is enhanced compared to the bulk mainly due to an increase of the d-state contribution.

The total energy difference $\Delta E_{(\downarrow \uparrow-\uparrow \uparrow)}(71 \mathrm{meV} /$ atom $)$ is positive and of the same order of magnitude as the result of the $4 \mathrm{f}$-core model [14]. The results of the present $\mathrm{LDA}+\mathrm{U}$ calculations for both bulk $\Delta E_{(A F M-F M)}(63$ meV/atom [16] - the difference in energies between bulk AFM and FM spin configurations) and surface $\Delta E_{(\downarrow \uparrow-\uparrow \uparrow)}$ (71 meV/atom) are in reasonable agreement with the results of $4 \mathrm{f}$-core model calculations $(85 \mathrm{meV} /$ atom for the bulk and $95 \mathrm{meV} /$ atom for the surface) 14. It shows that parallel coupling between surface and bulk magnetization is energetically preferable and there is no antiparallel surface-to-bulk magnetic coupling for the Gd surface. This conclusion is consistent with experimental observations of the in-plane component of surface layer magnetization to be parallel to the bulk [6].

The electron density of states (DOS) for the case of (energetically preferred) $\uparrow \uparrow$ coupled surface layer are shown in Fig. 1. There is a $4.5 \mathrm{eV}$ downward shift of the majority spin $4 \mathrm{f}$ states and a $1.5 \mathrm{eV}$ upward shift of minority spin $4 \mathrm{f}$ states compared to the LDA calculation results. This latter shift makes the minority spin $4 \mathrm{f}$ band practically empty and corrects the fundamental error of LDA 4f-band model. The exchange splitting of $4 \mathrm{f}$ states is enhanced in $\mathrm{LDA}+\mathrm{U}$ calculations by the amount of the Hubbard U [16] resulting in an $11 \mathrm{eV}$ splitting of majority and minority $4 \mathrm{f}$ states, close to the experimentally derived value $(12 \mathrm{eV})$ [3]. The formation of a surface state at the Gd surface clearly shows up as a peak of DOS in the vicinity of Fermi level (cf. Fig. 1) due to majority d states. From the DOS it is clear that LDA+U show 
strongly localized character of 4 f-states for both bulk and surface. However, the response of the $4 \mathrm{f}$ states to their environment does not allow them to be considered as a true core states.

In order to check numerical convergence of our results with respect to k-space integration, we increased the number of special k-points in the irreducible part of 2D BZ 22 from 32 to 50 in self-consistent calculations and found very little change in magnetic moment for both $\downarrow \uparrow$ and $\uparrow \uparrow$ magnetic configurations $\left(\leq 0.04 \mu_{B}\right)$. The calculated total energy difference $\Delta E_{(\downarrow \uparrow-\uparrow \uparrow)}=72 \mathrm{meV}$ per surface atom agrees very well with its value of $71 \mathrm{meV}$ for a smaller number of $\mathrm{k}$-points.

3.Strain Relaxation and Magnetic Ordering at the Gd Surface. LEED measurements [25] show that there is atomic structural relaxation near the Gd surface: the interlayer distance between surface and sub-surface Gd layers is about $2.6 \%$ smaller than its bulk value and the subsurface-to-bulk layer distance is about $1 \%$ bigger than its bulk value. We have performed $\mathrm{LDA}+\mathrm{U}$ calculations with the surface and sub-surface layers (i) with interlayer distances taken from the experiment [25] and (ii) with interlayer relaxations taken to be half way between the experimental surface values and the bulk values. As in the case of an ideal Gd surface we have considered two possible magnetic configurations $(\uparrow \uparrow, \downarrow \uparrow)$ with the surface layer coupled parallel and antiparallel to the FM bulk Gd. Here, 50 special k-points in the irreducible 1/3 part of the $2 \mathrm{D} \mathrm{BZ}$ were used.

The surface relaxation affects very little the values of the magnetic moment in comparison with the ideal surface: for both cases of $\uparrow \uparrow$ and $\downarrow \uparrow$ coupled surface layer there is a slight decrease in the values of the surface and sub-surface layers magnetic moment due to the change of conduction band magnetization caused by reduced interlayer distance.

There is, on the other hand, a surprisingly large enhancement of the magnetic coupling energy $\Delta E_{(\downarrow \uparrow-\uparrow \uparrow)}$ (cf., Table III) due to the surface relaxation: the energy difference increases by $90 \%$ in the comparison with the unrelaxed structure.

As was already mentioned, there is considerable experimental evidence of $T_{c}$ enhancement at the Gd surface. Since 4f-magnetic moments are well localised and interact due to RKKY-type of exchange interactions, the use of the Heisenberg-type Hamiltonian for the dependence of energy on spin configuration is physically justified for $\mathrm{Gd}$. For the sake of simplicity, we neglect the long-range behavior of exchange interactions for the Gd bulk and surface, assuming that significant physics can be discussed in terms of nearest-neighbor (NN) interactions and neglect anisotropy in exchange interaction between a $\mathrm{Gd}$ atom and its six in-plane and six interplane NN in the bulk and three interplane NN at the surface. The spin Hamiltonian is then given by:

$$
H=-B_{0} \sum_{i} \hat{S}_{i}-\sum_{i} \sum_{\delta} J_{i, i+\delta} \hat{S}_{i} \hat{S}_{i+\delta}
$$

where, $B_{0}$ is an external field, $J_{i, i+\delta}$ is an exchange coupling constant between the spin $i$ and its $\delta \mathrm{NN}\left(J^{b}\right.$ in the bulk and $J^{s}$ at the surface) and $\hat{S}_{i}$ is a spin operator. We then apply "molecular field" theory 26 to Eq.(1). It leads to different molecular fields acting on the spin at the surface and in the bulk, due to the different number of the interplane NN (six in the bulk, three at the surface), and the difference between bulk and surface exchange coupling constants $J_{b}$ and $J_{s}$. In the vicinity of the Curie temperature, when the value of the average spin moment $\langle S(T)>$ is small, it is possible to introduce a Landau-Ginzburg type model for the temperature dependence of $\langle S(T)>27$ using the continuum limit of the molecular field theory. Applying the procedure of Ref. 27 to the case of the hcp(0001) surface, we obtain the result that, for

$$
1.5-2 \frac{J_{b}}{J_{s}} \geq 0 \text { or } \frac{J^{s}}{J^{b}} \geq 4 / 3
$$

there is in addition to the bulk Curie temperature $T_{c}^{b}$ a surface Curie temperature $T_{c}^{s}$ which is connected to the bulk $T_{c}$ as:

$$
T_{c}^{s}=\left[1+\left(1.5-2 \frac{J_{b}}{J_{s}}\right)^{2}\right] T_{c}^{b} ; T_{c}^{b}=\frac{12 J_{b} S(S+1)}{3 k_{B}}
$$

The ratio $\Delta E_{(\downarrow \uparrow-\uparrow \uparrow)} / \Delta E(A F M-F M)$ (cf., Table II $)$ is then used to determine $J^{s} / J^{b}$ in Eq.(2) [28]. In the case of an ideal surface the condition Eq.(2) is not satisfied and there is no additional $T_{c}^{s}$. However, when the surface relaxation is taken into account, the condition Eq.(2) is satisfied and Eq.(3) yields $T_{c}^{s}=1.33 T_{c}^{b}$ in very good quantitative agreement with the recent experimental data [9] $\left(T_{c}^{s} \approx 1.29 T_{c}^{b}\right)$.

To summarize, we have presented the results of one of the first applications of the LDA $+\mathrm{U}$ total energy method to study the magnetic and electronic properties of a correlated metal. We have found that the use of $\mathrm{LDA}+\mathrm{U}$ instead of LDA yields FM alignment between surface and bulk magnetic moments, in agreement with experiment. An interlayer surface-to-bulk effective exchange coupling is calculated to be close to its bulk value for an ideal surface, but is enhanced by $90 \%$ by surface relaxation. This enhancement is sufficiently strong to produce an elevated Curie temperature at the surface, as observed experimentally. These results also have important implications for other rare earth (and possible also transition metal) surfaces and interfaces, and suggest that both $4 \mathrm{f}$ (and 3d) correlation and atomic structure must be accurately accounted for in order to quantitatively describe magnetism.

This research was supported by National Science Foundation Grant DMR-9802076 and U. S. Dept. of Energy, under Contract No. DE-AC03-76F00098. 
[1] J. Jensen and A. R. Mackintosh, Rare Earth Magnetism, Clarendon Press, Oxford 1991, 403.

[2] L. Roeland et al., J. Phys. F 5, L233 (1975).

[3] P. A. Dowben, D.N. McIlroy and D. Li, in Handbook on the Physics and Chemistry of Rare Earths, Elsevier North-Holland, NY 1997, 241.

[4] (a) C. Rau and M. Robert, Phys. Rev. Lett. 58, 2714 (1987); (b) C. Rau, J. Magn. Magn. Mater. 31-34, 874 (1983); (c) Y. A. Mamaev et al., Sov. Phys. Tech. Phys. Lett. 13, 642 (1987).

[5] D. Weller, S. F. Alvarado, M. Gudat, K. Schroder, M. Campagna, Phys. Rev. Lett. 54, 1555 (1985).

[6] G. A. Mulhollan, K. Garrison, J.L. Erskine, Phys. Rev. Lett. 69, 3240 (1992).

[7] H. Tang et al., Phys. Rev. Lett. 71, 444 (1993).

[8] D. Li et al., J. Phys. : Cond. Matter 4, 3929 (1992).

[9] E. D. Tober et al., Phys. Rev. Lett. 81, 2360 (1998).

[10] J. O. Dimmock and A. J. Freeman, Phys. Rev. Lett. 13, 750 (1964).

[11] J. Sticht and J. Kübler, Solid State Comm. 53, 529 (1985); M. Richter and H. Eschrig, Solid State Comm. 72, 529 (1989).

[12] D.J. Singh, Phys. Rev. B 44, 7451 (1991).

[13] B. N. Harmon, V. P. Antropov, A. I. Liechtenstein, I. V. Solovyev and V. I. Anisimov, J. Phys. Chem. Solids 56, 1521 (1995).

[14] O. Eriksson et al., Phys. Rev. B 52, 4420 (1995).

[15] A. I. Liechtenstein V.I. Anisimov and J. Zaanen, Phys. Rev. B 52, R5467 (1995).

[16] A. B. Shick, A. I. Liechtenstein, W. E. Pickett, Phys. Rev. B 60, October 15 (1999), cond-mat/9903439.

[17] R.Q. Wu, C.L. Fu, A.J. Freeman, Phys. Rev. B 44, 9400 (1991).

[18] E. Wimmer, H. Krakauer, M. Weinert, and A. J. Freeman, Phys. Rev. B 24, 864 (1981).

[19] A. B. Shick, D. L. Novikov, A. J. Freeman , Phys. Rev. B 57 R14259, (1997).

[20] B. J. Beandry and K. A. Gschneider, Jr., in Handbook on the Physics and Chemistry of Rare Earths, Elsevier North-Holland, NY 1982, 1216.

[21] H. J. Monkhorst and J. D. Pack, Phys. Rev. B 13, 5188 (1976).

[22] The symmetry was chosen to be consistent with the proper magnetic symmetry in the presence of spin-orbit coupling. It allows us to do relativistic self-consistent calculations starting from self-consistent scalar-relativistic effective potential and exchange-correlation field.
[23] S. V. Vonsovskii, Soviet Physics, JETP 16, 981 (1946).

[24] W. Nolting, T. Dambeck, G. Borstel, Z. Phys. B 94, 409 (1994).

[25] J. Giergiel et al., Phys. Rev. B 51, 10201 (1995).

[26] H. E. Stanley, Introduction to phase transitions and critical phenomena, Clarendon Press, Oxford 1971, 304.

[27] D. L. Mills, Phys. Rev. B 3, 3887(1971).

[28] We expect the exchange interaction in hcp Gd to be isotropic for nearest neighbors, whether in-plane or interplane, and this is justified from both bulk spin wave measurements (P-A. Lindgard, Phys. Rev. B 17, 2348 (1978)) and other bulk and surface calculations not shown here (A. B. Shick, W. E. Pickett, C.S. Fadley, (unpublished)).

TABLE I. Spin magnetic moments $\left(M_{s}\right.$ in $\left.\mu_{B}\right)$ for a $\mathrm{Gd}$ film with surface layer coupled parallel to the FM bulk resulting from scalar-relativistic $\mathrm{LDA}+\mathrm{U}$ calculations with experimental lattice constants. For "muffin-tin" (MT), these values are integrals over a "muffin-tin" sphere of radius 3.2

\begin{tabular}{lcccccc} 
a.u. & \multicolumn{7}{l}{ lotal } \\
\hline \hline$M_{s}$ & layer & $\mathrm{s}$ & $\mathrm{p}$ & $\mathrm{d}$ & $\mathrm{f}$ & to. \\
MT & $\mathrm{C}$ & 0.016 & 0.079 & 0.46 & 6.97 & 7.536 \\
$\mathrm{MT}$ & $\mathrm{S}-2$ & 0.019 & 0.081 & 0.49 & 6.97 & 7.576 \\
MT & $\mathrm{S}-1$ & 0.011 & 0.093 & 0.50 & 6.97 & 7.588 \\
MT & $\mathrm{S}$ & 0.041 & 0.074 & 0.67 & 6.975 & 7.773 \\
\hline Interstitial: & 2.076 & Vacuum: & 0.088 & \\
\hline \hline
\end{tabular}

TABLE II. Total energy difference between two magnetic configurations with the surface layer magnetically coupled antiparallel $(\downarrow \uparrow)$ and parallel $(\uparrow \uparrow), \quad \Delta E_{(\downarrow \uparrow-\uparrow \uparrow)}$ (meV/atom), for a Gd surface with (a) an ideal bulk atomic structure (b) a relaxed structure from experiment and (c) an "average" (between bulk and relaxed surface) values of surface-to-subsurface-to-bulk distances and its ratio to the total energy difference between AFM and FM bulk $(\triangle E(A F M-F M)=63 \mathrm{meV} /$ atom $)$ : $\Delta E_{(\downarrow \uparrow-\uparrow \uparrow)} / \Delta E(A F M-F M)$

\begin{tabular}{ccc}
\hline \hline & $\Delta E_{(\downarrow \uparrow-\uparrow \uparrow)}$ & $\Delta E_{(\downarrow \uparrow-\uparrow \uparrow)} / \Delta E(A F M-F M)$ \\
$\mathrm{a}$ & 72 & 1.14 \\
$\mathrm{~b}$ & 135 & 2.14 \\
$\mathrm{c}$ & 136 & 2.16 \\
\hline \hline
\end{tabular}



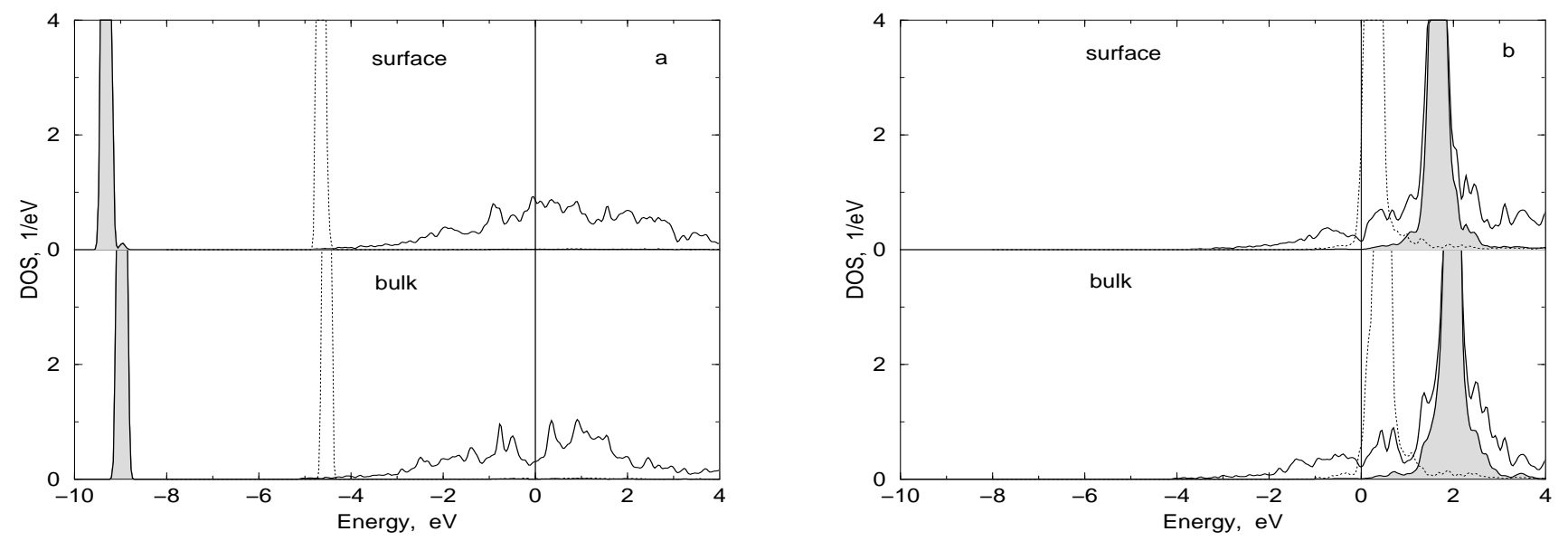

FIG. 1. DOS for Gd-film : LDA+U spin-up (a); spin-down (b), 4f-states (filled); LDA 4f-states (dotted) 